\title{
Fundamental Limitation of van der Waals Homoepitaxy by Stacking Fault Formation in WSe2
}

Wouter Mortelmans ${ }^{1,2}$, Ankit Nalin Mehta ${ }^{2,3}$, Yashwanth Balaji ${ }^{2,4}$, Salim El Kazzi ${ }^{2}$, Stefanie Sergeant $^{2}$, Michel Houssa ${ }^{3}$, Stefan De Gendt ${ }^{2,5}$, Marc Heyns ${ }^{1,2}$ and Clement Merckling ${ }^{2}$

\footnotetext{
${ }^{1}$ KU Leuven, Department of Materials Engineering, Kasteelpark Arenberg 44, 3001 Leuven, Belgium ${ }^{2}$ Imec, Kapeldreef 75, 3001 Leuven, Belgium

${ }^{3}$ KU Leuven, Department of Physics and Astronomy, Celestijnenlaan 200d, 3001 Leuven, Belgium

${ }^{4}$ KU Leuven, Department of Electrical Engineering, Kasteelpark Arenberg 10, 3001 Leuven, Belgium

${ }^{5} \mathrm{KU}$ Leuven, Department of Chemistry, Celestijnenlaan 200f, 3001 Leuven, Belgium
}

\begin{abstract}
:
As interest in layered van der Waals (vdW) materials keeps increasing, fundamental knowledge about their synthesis is gaining more and more value. The defect-free heteroepitaxial integration of $\mathrm{vdW}$ materials on large-area substrates is currently thoroughly being researched since it might encompass a successful transition of these materials to industrial applications. To date, Transition Metal Dichalcogenides (TMDs) are considered as one of the most promising $\mathrm{vdW}$ materials within the field of nanoelectronics. Nevertheless, the electrical characterization of heteroepitaxially grown TMDs still shows inferior performance as compared to exfoliated TMD flakes. This is mainly attributed to the high density of defects resulting from their challenging vdW heteroepitaxial synthesis. In this work, we have investigated in depth the vdW homoepitaxial synthesis of the $\mathrm{WSe}_{2} \mathrm{TMD}$ compound. We have demonstrated that even for homoepitaxy, the simplest type of crystal growth, challenges such as the formation of $60^{\circ}$ twins need to be addressed. We evidenced the presence of $60^{\circ}$ twins during vdW homoepitaxy which is assigned to stacking faults. The formation of these stacking faults is associated with their very similar binding energy as revealed by Density Functional Theory (DFT) calculations. Therefore, stacking faults are identified in this work to be the fundamental limitation of lowly-defective TMD vdW epitaxy. Furthermore, a generalized model is developed that determines the lower limit of the defect density based on the degree of control on the bilayer stacking phase and the nucleation density of the TMD compound. This model therefore assesses and quantifies for the first time the ultimate defect density level that can be achieved with vdW epitaxially grown 2D materials.
\end{abstract}

KEYWORDS: Molecular Beam Epitaxy, van der Waals epitaxy, Transition Metal Dichalcogenides, $\mathrm{WSe}_{2}$, homoepitaxy, stacking faults, DFT. 


\section{INTRODUCTION}

Thanks to the finding and the subsequent widespread research on graphene ${ }^{1}$, interest in van der Waals (vdW) materials has increased expressively for the purpose of future semiconductor technologies ${ }^{2,3}$. Like graphene, vdW materials are characterized by strong covalent intralayer bonding in coexistence with weak vdW interlayer bonding. A very promising class of vdW materials are the Transition Metal Dichalcogenides (TMDs) ${ }^{4}$. One monolayer (ML) of such a $\mathrm{vdW}$ material is composed of a layer of metal atoms (M) covalently sandwiched between two layers of chalcogen atoms $\left(\mathrm{X}_{2}\right)$. Accordingly, they are classified as $\mathrm{MX}_{2}$ where they can exist in various compounds such as $\mathrm{WSe}_{2}, \mathrm{MoS}_{2}, \mathrm{HfTe}_{2}, \ldots 5$ Unlike graphene, TMDs are characterized by the presence of a bandgap within a desirable energy range $(0.5-2.0 \mathrm{eV})^{6,7}$ which makes them promising material candidates for (opto-)electronic applications ${ }^{8-11}$. In addition, the recent observation of Weyl fermions ${ }^{12}$, massless Dirac fermions ${ }^{13}$ and chargedensity waves ${ }^{13}$ in various phases of TMD compounds expand the potential application even further.

Regarding the epitaxy of these materials, the specific feature of the weak vdW interlayer interactions has important implications. Pioneering work on vdW heteroepitaxy has learned that due to the absence of dangling bonds, the interactions at the heterointerface are governed by weaker vdW interactions as compared to the strong covalent interactions for conventional heteroepitaxy ${ }^{14}$. This aspect of vdW epitaxy generally results in relaxed lattice matching requirements which makes these materials even more promising for nanoelectronic applications ${ }^{15}$. Indeed, unique properties can now be engineered by stacking various vdW layers on top of each other without critical lattice matching or defect formation caused by strain relaxation $^{16,17}$.

Weak vdW interlayer interactions however also induce negative aspects regarding the heteroepitaxy. This can be seen from the difficult enforcement of a unique in-plane epitaxial relation between the vdW layer and the growth substrate ${ }^{18-20}$. Recently, the engineering of the interfacial interactions during vdW heteroepitaxy has demonstrated to advance the integration of vdW materials onto commercial substrates ${ }^{21,22}$. Nonetheless, additional challenges such as the relatively large in-plane mosaicities ${ }^{23-25}$ and the continuous presence of $60^{\circ}$ twins $^{26-28}$ are also to be resolved. As for the former, yet no clear solution but increasing the growth temperature is proposed ${ }^{29}$, the latter might be improved by enforcing step-edge guided nucleation $^{30,31}$ or clever surface engineering ${ }^{32,33}$. On the other hand, even if the heteroepitaxy of TMDs is revealed to be very challenging, no studies were done until now on the homoepitaxy of TMD compounds due to the nonexistence of TMD substrates. In this work, we propose to 
fabricate virtual TMD substrates to better understand the vdW nucleation and growth behavior in the simple case of TMD vdW homoepitaxy. This, to better address the twinning/stacking control that the research community is currently facing during vdW heteroepitaxy processes.

\section{RESULTS AND DISCUSSION}

In this work, the crystal growth of the $\mathrm{WSe}_{2} \mathrm{TMD}$ compound is in-depth investigated on a single crystalline $\mathrm{WSe}_{2}$ surface. The virtual $\mathrm{WSe}_{2}$ substrate is obtained by first performing an exfoliation of $2 \mathrm{D} \mathrm{WSe}_{2}$ flakes onto silicon substrates. This allows to study the nucleation and growth of $\mathrm{WSe}_{2}$ directly on single crystalline $\mathrm{WSe}_{2}(0001)$ surfaces (see Supporting Information). Subsequently, the virtual 2D substrates are inserted into the ultra-high vacuum molecular beam epitaxy chamber. The $\mathrm{WSe}_{2}$ homoepitaxy is then performed using the PlasmaAssisted Molecular Beam Epitaxy (PA-MBE) technique ${ }^{21,34}$. After the PA-MBE growth, the samples are immediately ex-situ characterized by Atomic Force Microscopy (AFM) and PlaneView Transmission Electron Microscopy (PV-TEM).

\subsection{AFM and crystal analysis}

The homoepitaxy of $\mathrm{WSe}_{2}$ is characterized using AFM and algorithmically analyzed in Figure 1. Figure 1a represents the AFM image of $\sim 0.3 \mathrm{ML}$ of $\mathrm{WSe}_{2}$ homoepitaxy with optimal growth conditions at $450{ }^{\circ} \mathrm{C}$ and $0.15 \mathrm{ML} \mathrm{h}^{-1}$ as attested by the well-defined equilateral triangular shape of the grown $\mathrm{WSe}_{2}$ crystals. The nominal growth is limited to $\sim 0.3 \mathrm{ML}$ in order to prevent the coalescence process as to better highlight the shape, size and orientation of the initial $\mathrm{WSe}_{2}$ crystals. The height histogram of the corresponding AFM image is presented in Figure 1b. The peak centered around the lowest heights corresponds to the pixels originating from the $\mathrm{WSe}_{2}$ flake surface while the peak at higher heights corresponds to the pixels from the $\mathrm{MBE} \mathrm{WSe}_{2}$ crystals. The histogram emphasizes a clear separation between the $\mathrm{WSe}_{2}$ epilayer and the $\mathrm{WSe}_{2}$ virtual substrate. This, despite the very small thickness of the grown ML-thick WSe 2 crystals $(\sim 0.6 \mathrm{~nm})$. The nucleation density is calculated to be as large as $\sim 5 \mathrm{E}+10 \mathrm{~cm}^{-2}$.

An algorithm that senses the characteristic equilateral triangular shape of the grown $\mathrm{WSe}_{2}$ crystals is developed and applied for the supporting quantitative analysis of the AFM image of Figure 1a. When a certain $\mathrm{WSe}_{2}$ crystal is detected having an equilateral triangular shape, the algorithm can accurately extract crystal properties such as the crystal's grain size ' $a$ ' and its azimuthal in-plane alignment ' $\alpha$ ', as defined and illustrated in Figure 1c. The algorithmic analysis is therefore able to construct the triangular grain size distribution (Figure 


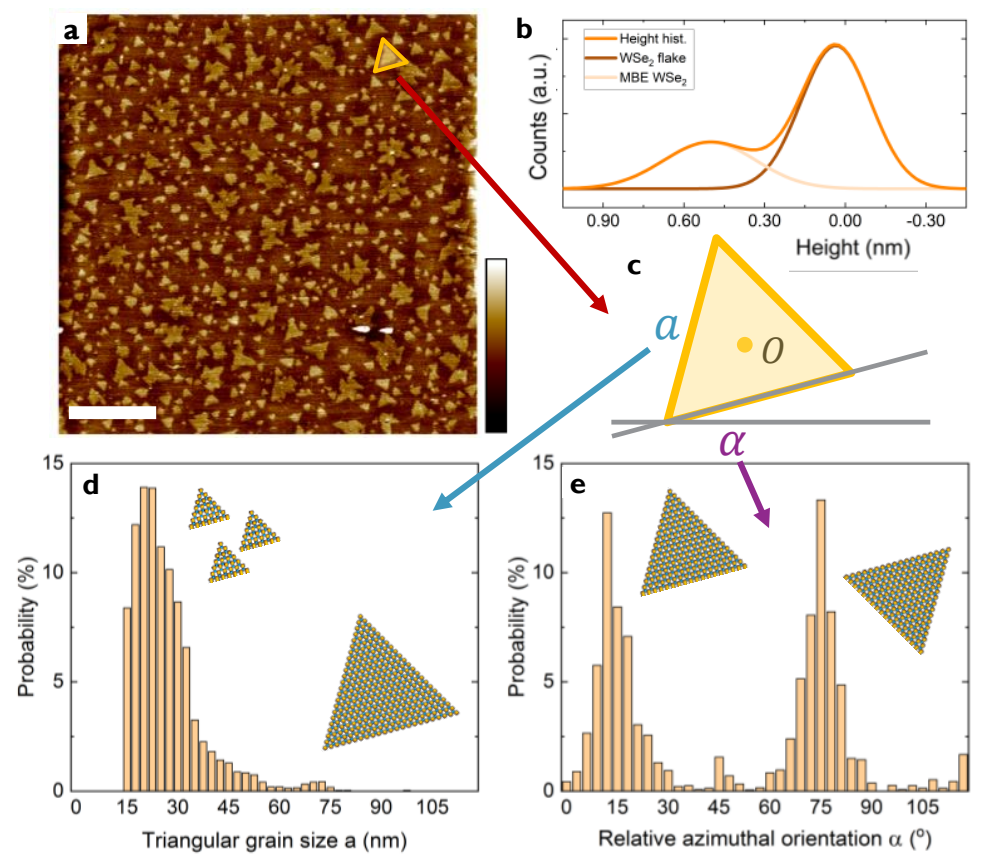

Figure I: Homoepitaxy of WSez characterized by AFM and quantitatively analyzed. a) AFM image of WSez nucleated and grown on the single crystalline WSe2 surface. Scale bar is $200 \mathrm{~nm}$. Height scale is $2 \mathrm{~nm}$. b) Height histogram of the AFM image presented in (a), fitted with two Gaussians corresponding to the WSe flake and the MBE-grown WSe2. c) Illustration of the characteristic equilateral triangular shape of the $\mathrm{WSe}_{2}$ crystals, defining the triangular grain size and relative azimuthal orientation respectively noted by 'a' and ' $\alpha$ '. ' $O$ ' corresponds to the center of mass of the crystal. $d$ ) Triangular grain size distribution of the WSe 2 homoepitaxy obtained from the algorithmic crystal analysis of the presented AFM image in (a). e) Distribution of the relative in-plane azimuthal orientation of the $\mathrm{WS} e_{2}$ crystals for the WSe homoepitaxy, obtained from the algorithmic crystal analysis of the presented AFM image in (a).

1d) and the relative azimuthal orientation distribution (Figure 1e) of the $\mathrm{WSe}_{2}$ homoepitaxy which capture important insights.

The grain size distribution of the equilateral triangles is presented in Figure 1d and is quantified to be skewed Gaussian. This originates from the coexistence of a high density of small triangles $(\sim 20 \mathrm{~nm})$ together with a lower density of larger triangles. It indicates that for the time frame studied (1) a saturated nucleation density is not yet reached and that (2) spontaneous Ostwald ripening is not observed for this epitaxial system. Both these observations could be explained by the small adatom diffusion length of the involved atomic species in the epitaxial process ${ }^{35}$. The minimal grain size for proper triangular crystal detection was set at 15 $\mathrm{nm}$. The largest grain size of uncoalesced triangles (for a nominal growth of only $\sim 0.3 \mathrm{ML}$ ) is probed at $\sim 80 \mathrm{~nm}$. This is similar to literature results of MBE-based TMD heteroepitaxies performed on 2D crystal surfaces ${ }^{35-39}$.

The degree of in-plane epitaxial alignment is quantified relying on the distribution of the relative azimuthal orientation of the triangular crystals. This distribution is presented in Figure 1e. A clear preferential in-plane alignment is observed from the histogram which indicates the good homoepitaxial registry of the MBE-grown $\mathrm{WSe}_{2}$ crystals with the $\mathrm{WSe}_{2}$ 
substrate. Two distinct peaks are clearly noticed in the presented histogram, originating from the large probability to find equilateral triangular crystals with a relative azimuthal orientation at respective angles of $15^{\circ}$ and $75^{\circ}$. Surprisingly, the peaks are separated from each other by $60^{\circ}$ which indicates that the homoepitaxy is characterized by a 6-fold in-plane rotational symmetry. The lack of in-plane inversion symmetry that makes TMDs however only 3-fold symmetric in-plane ${ }^{21}$, will as a consequence have a significant impact on the defect density of the grown homoepitaxial $\mathrm{WSe}_{2}$ layer.

\subsection{TEM and stacking analysis}

The homoepitaxial stack is picked-up and transferred to the TEM grid for subsequent structural analysis. This study is presented in Figures $2 \mathrm{a}$ and $2 \mathrm{~b}$ and is once more supported by a quantitative analysis relying on the developed algorithm (Figures $2 \mathrm{f}$ and $2 \mathrm{~g}$ ). Figure $2 \mathrm{a}$ represents the Selected Area Electron Diffraction Pattern (SAEDP) of the $\mathrm{WSe}_{2}$ nucleated and grown on a single crystalline $\mathrm{WSe}_{2}$ bilayer. The single-dot-like hexagonal diffraction pattern confirms the perfect epitaxial registry of the $\mathrm{WSe}_{2}$ crystals with the surface of the $\mathrm{WSe}_{2}$ flake. This is in good agreement with the observed in-plane distribution of the $\mathrm{WSe}_{2}$ crystals as quantified by the algorithmic analysis on the AFM image (Figure 1e). It also confirms the absence of any strain in the grown $\mathrm{WSe}_{2}$ likely originating from the absence of lattice mismatch between the $\mathrm{WSe}_{2}$ epilayer and $\mathrm{WSe}_{2}$ virtual substrate.

A color-coded Dark-Field (DF-)TEM image that is sensitive to the $\mathrm{WSe}_{2}$ bilayer stacking configuration is presented in Figure $2 \mathrm{~b}$. The construction of this image is thoroughly explained in the Supporting Information based on the homoepitaxy of $\mathrm{WSe}_{2}$ on a $\mathrm{WSe}_{2}$ singlelayer. Three different bilayer stacking configuration are differentiated from the color-coded DF-TEM image: 2H:AA', 3R:AB and 3R:AC. They are respectively presented in Figures 2cd-e using cross-sectional ball-and-stick schematics in agreement with their occurrence as highlighted by the black line in Figure 2b. The 2H:AA' bilayer stacking configuration has simultaneously the chalcogen and metal atoms of the ML layer stacked on respectively the metal and chalcogens atoms of the bottom ML. This is only enabled by a $60^{\circ}$ in-plane rotation of one ML with respect to the other. A bilayer that is stacked in the 3R phase lacks this relative $60^{\circ}$ in-plane rotation and is therefore only able to stack the metal atoms of one ML on top of the chalcogen atoms of the other, or vice versa. Accordingly, the 3R:AB bilayer stacking configuration has the chalcogen atoms of the top ML stacked on the metal atoms of the bottom ML, whereas the 3R:AC configuration has the metal ones stacked on the chalcogens. The 

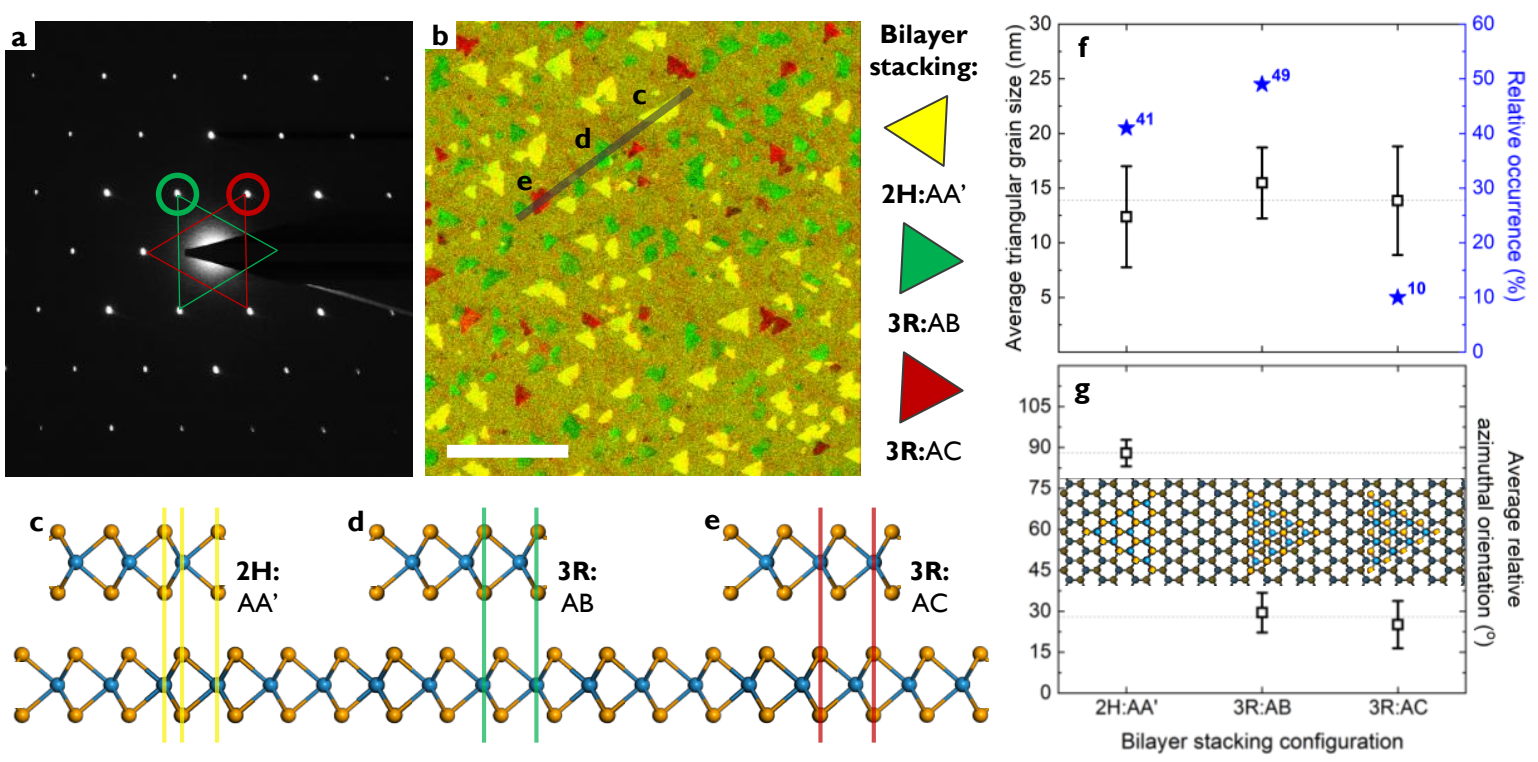

Figure 2: Homoepitaxy of WSez characterized by TEM and quantitatively analyzed. a) SAEDP of the WSez nucleated and grown on the single crystalline WSe 2 bilayer. The green and red triangles assemble the diffractions spots that correspond to the different $\{\overline{\mathbf{1}} \mathbf{1 0 0}\}$ families. The encircled green and red diffraction spots are used for the reconstruction of the image presented in (b). b) Color-coded DF-TEM image of the WSe2 homoepitaxy. The different colors represent the various bilayer stacking configurations. The yellow color corresponds to the $2 \mathrm{H}: \mathrm{AA}^{\prime}$ configuration. The green and red colors correspond to respectively the $3 R: A B$ and $3 R: A C$ bilayer stacking configuration. Scale bar is $100 \mathrm{~nm}$. c, d, e) Cross-sectional schematic representations of respectively the $2 \mathrm{H}: A A^{\prime}, 3 \mathrm{R}: \mathrm{AB}$ and $3 \mathrm{R}: \mathrm{AC}$ bilayer stacking configurations. $f$ ) Correlation between the average triangular grain size (left axis) and the bilayer stacking configuration. The relative occurrence of the stacking configuration is presented on the right axis in blue. g) Correlation between the average relative in-plane azimuthal orientation of the MBE-grown WSe2 crystals and the bilayer stacking configuration. Top-view schematic representations of the various bilayer stacking configurations are shown in the center of the graph.

presented color-coded DF-TEM image enables to study the $\mathrm{WSe}_{2}$ crystal grain size and its inplane alignment in function of the type of bilayer stacking configuration. The quantitative values that are obtained from this analysis are discussed in the next paragraphs and summarized in Table S1 in the Supporting Information. As will follow, this study will enable an appropriate quantification of the defect density which remained to date highly challenging for $2 \mathrm{D} v \mathrm{vW}$ epitaxial systems.

The impact of the bilayer stacking configuration on the average triangular grain size is presented in Figure 2f. As seen from this figure, it is found that the average triangular grain size is similar for all the three observed stacking configurations. The calculated average grain sizes are $12.4 \pm 4.6,15.5 \pm 3.2$ and $13.9 \pm 5.0 \mathrm{~nm}$ for respectively the $2 \mathrm{H}: \mathrm{AA}, 3 \mathrm{R}: \mathrm{AB}$ and $3 \mathrm{R}: \mathrm{AC}$ bilayer stacking configurations. They demonstrate that the growth rate of the $\mathrm{WSe}_{2}$ crystals during vdW homoepitaxy is independent from the bilayer stacking configuration. The average crystal growth rate (defined as the average growth rate of every nucleated crystal) is estimated to be as low as $13 \pm 2.4$ adatoms $\min ^{-1}$ for $\mathrm{W}$ and $26 \pm 4.8$ adatoms $\min ^{-1}$ for Se. 
Unlike the average $\mathrm{WSe}_{2}$ triangular crystal growth rate, the average $\mathrm{WSe}_{2}$ nucleation rate shows a clear dependency on the bilayer stacking configuration. This is seen from the differences in relative occurrence of the various stacking configurations and is presented in blue on the right $\mathrm{Y}$-axis of Figure $2 \mathrm{f}$. A relative occurrence of $41 \%, 49 \%$ and $10 \%$ is respectively measured for the $2 \mathrm{H}: \mathrm{AA}^{\prime}, 3 \mathrm{R}: \mathrm{AB}$ and $3 \mathrm{R}: \mathrm{AC}$ bilayer stacking configuration. These correspond to respective average nucleation rates of $5.4 \mathrm{E}+08,6.5 \mathrm{E}+08$ and $1.3 \mathrm{E}+08$ nuclei $\mathrm{cm}^{-2} \mathrm{~min}^{-1}$. The average nucleation rates of the $2 \mathrm{H}: \mathrm{AA}^{\prime}$ and the $3 \mathrm{R}: \mathrm{AB}$ configurations are calculated to be close to each other, while the nucleation rate of the 3R:AC configuration is significantly lower $(>x 4)$. Hence, not all bilayer stacking configurations are believed to be energetically equivalent. A more in-depth discussion on this will be presented later in the manuscript relying on Density Functional Theory (DFT) calculations.

The correlation between the in-plane alignment of the $\mathrm{WSe}_{2}$ crystals and the bilayer stacking configuration is highlighted in Figure $2 \mathrm{~g}$. Here, the average relative azimuthal orientation of the $\mathrm{MBE} \mathrm{WSe}_{2}$ crystals is presented in function of the bilayer stacking configuration. For the $2 \mathrm{H}: \mathrm{AA}^{\prime}$ configuration, an average in-plane angle of $88 \pm 5^{\circ}$ is calculated. The in-plane angles that are calculated for the 3R:AB and 3R:AC configurations are respectively $30 \pm 7^{\circ}$ and $25 \pm 9^{\circ}$. A relative in-plane azimuthal difference of $61 \pm 7^{\circ}$ is therefore calculated between the $2 \mathrm{H}$ and $3 \mathrm{R}$ bilayer stacked phases. This highlights that the MBE crystals that are stacked in the $2 \mathrm{H}$-phase are in-plane rotated by $60^{\circ}$ relative to the MBE crystals that are stacked in the 3R-phases (see top-view ball-and-stick schematics in Figure 2g).

The clear correlation between the in-plane azimuthal angle and the bilayer stacking configuration proves that the orientation of the $2 \mathrm{D}$ crystals and the stacking phase are clearly related one-to-one ${ }^{40}$. This is particularly helpful when one wants to distinguish both phases of the bilayer stacking ( $2 \mathrm{H}$ versus $3 \mathrm{R}$ ) without performing demanding and complex plane-view TEM analyses. A clear differentiation can now also be made from AFM analyses. In specific, the distribution of the in-plane alignment obtained from the AFM analysis that is presented in Figure 1e, can now link each individual peak to one specific phase of the bilayer stacking $(2 \mathrm{H} / 3 \mathrm{R})$. Once more, this highlights that both the $2 \mathrm{H}$ and $3 \mathrm{R}$ bilayer phases are simultaneously formed during the $\mathrm{WSe}_{2}$ homoepitaxy.

\subsection{DFT calculations}

As discussed in Section 2.2, our TEM analysis suggested that not all bilayer stacking configurations have a similar average nucleation rate (see also Table S1 in the Supporting Information). The 3R:AB bilayer stacking configuration was found to have the highest 
nucleation rate closely followed by the $2 \mathrm{H}: \mathrm{AA}^{\prime}$ configuration. The 3R:AC configuration was found to have a significantly lower nucleation rate which as will follow might be explained by the presence of defects in the $\mathrm{WSe}_{2}$ starting surface such as Se vacancies ${ }^{41}$. To elaborate further on this, various bilayer stacking binding energies are calculated relying on ab-initio calculations $^{42}$ (Figure 3a). These binding energies are calculated for the case of a completely defect-free $\mathrm{WSe}_{2}$ bilayer stack (black circles Figure 3a) and for a stack having Se vacancies in the upper chalcogen plane of the bottom ML (blue stars Figure 3a). The impact of other type of defects (such as Se or W vacancies in the top ML) on the binding energy can be found in the Supporting Information.

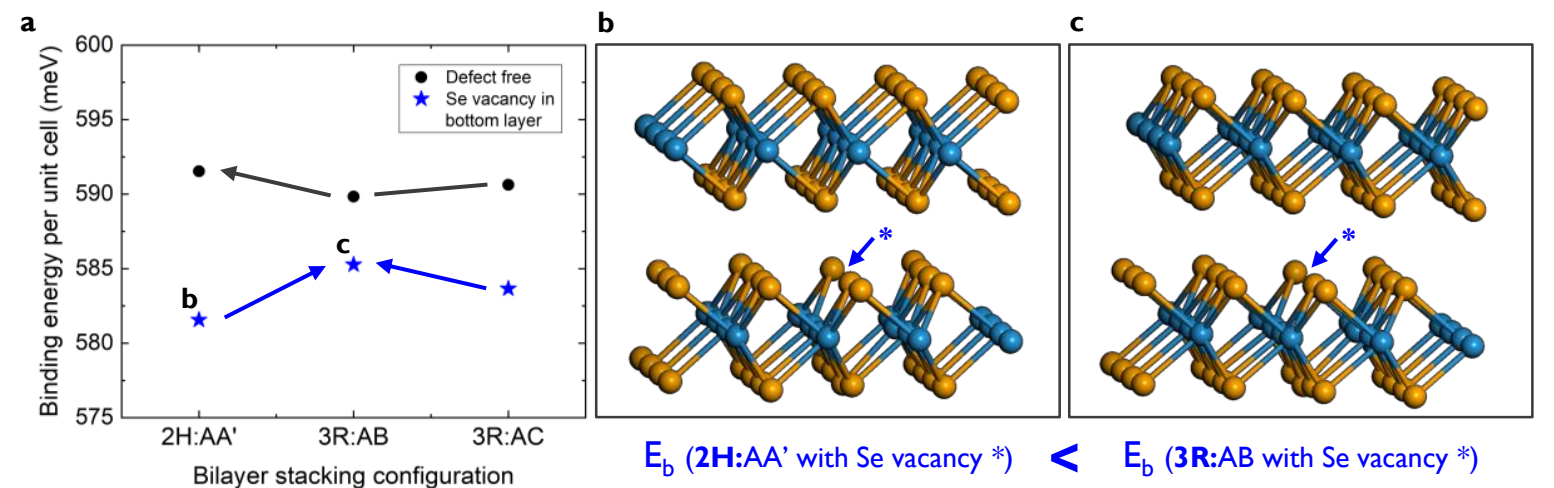

Figure 3: DFT calculations with corresponding relaxed structures. a) Calculated binding energies per unit cell of the WSe bilayer in function of the bilayer stacking configuration. The energies are calculated for the case of a defect-free bilayer (black circles) and for the case of a bilayer having Se vacancies in the upper plane of the bottom ML (blue stars). b) Tilted crosssectional ball-and-stick schematic of the relaxed WSe2 bilayer stacked in the $2 \mathrm{H}: \mathrm{AA}^{\prime}$ configuration having a Se vacancy in the top chalcogen plane of the bottom ML. c) Tilted cross-sectional ball-and-stick schematic of the relaxed WSez bilayer stacked in the 3R:AB configuration having a similar Se vacancy. The Se vacancies are highlighted with an asterisk.

The calculated binding energies of the defect-free bilayer stack reveal that the $2 \mathrm{H}: \mathrm{AA}^{\prime}$ configuration has a slightly larger binding energy as compared to the $3 \mathrm{R}: \mathrm{AB}$ and $3 \mathrm{R}: \mathrm{AC}$ configurations that both have a very similar binding energy. This implies that the $2 \mathrm{H}: \mathrm{AA}^{\prime}$ bilayer stacking configuration is energetically preferred compared to the other configurations and would therefore have the highest nucleation rate. However, since all calculated binding energies differ only by few meV per unit cell, the nucleation rates of both the $3 \mathrm{R}: \mathrm{AB}$ and 3R:AC configurations are only expected to be slightly lower as compared to the nucleation rate of the 2H:AA' configuration. Nonetheless, the nucleation rates that are obtained in our experiments do not completely follow the proposed trends as revealed by our calculations on a defect-free $\mathrm{WSe}_{2}$ bilayer stack. This explanation is therefore only believed to cover part of the phenomena that are observed. 
The plausible presence of Se vacancies at the $\mathrm{WSe}_{2}$ growth surface during the $\mathrm{WSe}_{2}$ homoepitaxy however, is found responsible for the shift in stacking energy that explains our experimental observations. The presence of these vacancies are predicted to severely decrease the binding energy of the $2 \mathrm{H}: \mathrm{AA}$ ' bilayer stacking configuration, and additionally to lift the degeneracy of the 3R:AB and 3R:AC configurations towards a higher binding energy for the 3R:AB bilayer stacking configuration. The presence of Se vacancies at the growth surface are therefore found to favor a higher nucleation rate of the $3 \mathrm{R}: \mathrm{AB}$ configuration as compared to the $2 \mathrm{H}: \mathrm{AA}$ ' configuration, and additionally as compared to the 3R:AC configuration. The relaxed simulated ( $4 \times 4 \times 1$ supercells) structures having one Se vacancy at the growth surface for the $2 \mathrm{H}: \mathrm{AA}^{\prime}$ and the 3R:AB bilayer stacking configuration are presented in Figures $3 \mathrm{~b}$ and $3 \mathrm{c}$ respectively using tilted cross-sectional ball-and-stick schematics. The combination of nucleation at both defect free areas (2H:AA' slightly preferred) and at areas with Se vacancies (3R:AB slightly preferred) can explain the experimentally observed trends in stackingdependent nucleation rate.

\subsection{Defect density model and calculation}

The simultaneous presence of various bilayer stacking configurations and consequently the lack of control on the bilayer stacking phase, even for the simplest type of crystal growth (i.e. homoepitaxy), is highlighted in this manuscript to be highly problematic. Indeed, the coalescence of crystals originating from both these dissimilar bilayer stacking phases will create defective $60^{\circ}$ Grain Boundaries (GBs) as a result of the corresponding 3-fold in-plane symmetry of the TMD compounds ${ }^{43,44}$. Therefore, the lack of control on the bilayer stacking phase will set a fundamental limitation on the minimal defect density of the grown TMD. Even more, the inability to control the stacking configuration might be typical for vdW materials in general, since weak vdW interlayer interactions are indeed expected to only weakly couple with the underneath substrate ${ }^{42}$.

The effect of the inability to control the bilayer stacking phase during the $\mathrm{WSe}_{2}$ homoepitaxy on the defect density is presented in Figure 4. When two $\mathrm{WSe}_{2}$ crystals coalesce during the homoepitaxial growth, a defective $60^{\circ} \mathrm{GB}$ is formed when both crystals are stacked in a various bilayer stacking phase ( $3 \mathrm{R}$ versus $2 \mathrm{H}$ ). This phenomenon is illustrated using HighResolution (HR)-STEM in Figure 4a. The green triangle represents the crystal that is stacked in the 3R:AB bilayer stacking configuration, whereas the yellow triangle represents the crystal stacked in the $2 \mathrm{H}: \mathrm{AA}^{\prime}$ configuration. Since they are stacked in a various phase, their 

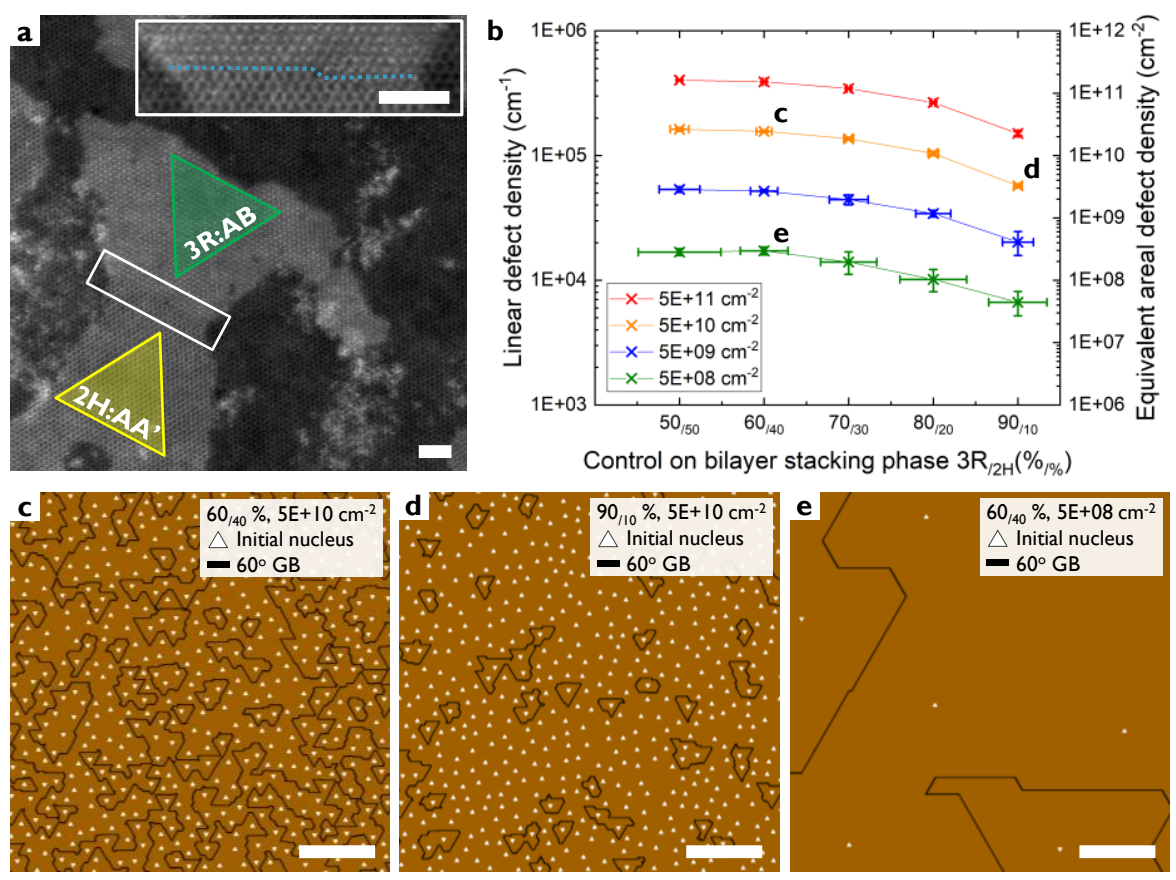

Figure 4: Defect density as a result of $60^{\circ}$ grain boundaries. a) HR-STEM image of the WSe $\mathrm{S}_{2}$ homoepitaxy. The green triangle highlights the WSe2 crystal that is stacked in the 3R:AB bilayer stacking configuration. The yellow triangle highlights the WSe2 crystal that is stacked in the $2 \mathrm{H}: \mathrm{AA}^{\prime}$ configuration. The coalescence of such two crystals results in the formation of a $60^{\circ}$ grain boundary as surrounded in the image with the withe rectangle. In the inset of (a), the enlarged image of the $60^{\circ}$ grain boundary is presented. The grain boundary is highlighted using a blue dashed line. Scale bar is $2 \mathrm{~nm}$. b) The calculated lower limit for the defect density of TMD epitaxy in function of the nucleation density and the control on the bilayer stacking phase. $O n$ the left $Y$-axis, the linear defect densities are presented per $\mathrm{cm}$. On the right $Y$-axis they are expressed in equivalent areal defect densities per $\mathrm{cm}^{2}$. c) Top-view representation of a fully closed WSe2 $M L$ with a nucleation density of $5 \mathrm{E}+10 \mathrm{~cm}^{-2}$ and a control on the bilayer stacking phase of $60 \% / 40 \%$. The combination of this specific nucleation density and bilayer staking control is highlighted in (b) using the letter ' $c$ '. The white triangles represent the initial nucleation sites and their in-plane azimuthal orientation correspond to a particular bilayer stacking phase. The triangles that are pointing upwards $(0 \circ)$ are stacked in the $3 \mathrm{R}$ phase while the triangles that are pointing downwards are stacked in the $2 \mathrm{H}$ phase $\left(60^{\circ}\right)$. The black lines correspond to the presence of $60^{\circ}$ grain boundaries. d) Similar representation as in (c) but with an improved control on the bilayer stacking phase $(90 / 10$ instead of $60 / 40)$, highlighted in (b) using the letter ' $d$ '. e) Similar representation as in (c) but with an improved nucleation density $\left(5 \mathrm{E}+08 \mathrm{~cm}^{-2}\right.$ instead of $\left.5 \mathrm{E}+10 \mathrm{~cm}^{-2}\right)$, highlighted in (b) using the letter 'd'.

coalescence will result in the formation of a $60^{\circ} \mathrm{GB}$. This coalescence process is highlighted with a white rectangle in Figure $4 \mathrm{a}$, where in the inset the $60^{\circ} \mathrm{GB}$ is marked with a blue dashed line. Accordingly, the $\mathrm{WSe}_{2}$ homoepitaxy that is characterized by a high nucleation density $\left(\sim 5 \mathrm{E}+10 \mathrm{~cm}^{-2}\right)$ in combination with a lack of control on the bilayer stacking phase $(60 \% / 40 \%$ $3 \mathrm{R}_{/ 2 \mathrm{H}}$ ) will result in a highly defective material synthesis with degraded electrical performances $^{45,46}$.

In order to quantify the defect density, the homoepitaxial nucleation and growth is modelled and the outcomes are presented in Figure $4 \mathrm{~b}$. This figure represents a lower limit for the total defect density of TMD epitaxy in function of the nucleation density and the control on the bilayer stacking phase. The defect densities are quantified for nucleation densities ranging from $\sim 5 \mathrm{E}+08$ to $\sim 5 \mathrm{E}+11 \mathrm{~cm}^{-2}$ and for a control on bilayer stacking ranging from $50_{/ 50}$ to $90_{/ 10}\left(3 \mathrm{R}_{/ 2 \mathrm{H}}\right)$. The linear defect densities (i.e. per $\mathrm{cm}$ ) are calculated along three symmetrical 
$\langle 1 \overline{1} 00\rangle$ in-plane directions and averaged. Additionally, the averaged linear defect densities are squared to equivalent areal defect densities expressed per $\mathrm{cm}^{2}$. A summary table of the specific examples that are discussed in the next paragraphs together with their corresponding defect densities is presented in the Supporting Information (Table S2). Note, that the quantified defect densities are an underestimation of the total defect density since the model only accounts for $60^{\circ} \mathrm{GBs}$ that are formed between the coalescence of crystals that are consecutively stacked in the $2 \mathrm{H}$ and $3 \mathrm{R}$ bilayer phases. The model therefore excludes point defects such as vacancies and defects that are formed between crystals that are stacked in the same phase but from a different configuration (e.g. 3R:AB versus 3R:AC). Nonetheless, the model represents and reveals novel understandings in the quantification of the defect density of TMD epitaxy, which remained to date highly unidentified.

The visual representations of the calculated defect densities are presented in Figures $4 \mathrm{c}$-d-e. These figures correspond to top-view images of fully closed $\mathrm{WSe}_{2} \mathrm{MLs}$. The initial nucleation sites and the bilayer stacking phases are indicated using a white triangle having a certain in-plane orientation $\left(0^{\circ}=3 \mathrm{R}\right.$ and $\left.60^{\circ}=2 \mathrm{H}\right)$. The presence of the $60^{\circ} \mathrm{GBs}$ resulting from the coalescence of crystals that are stacked in the various bilayer phases $(3 \mathrm{R} / 2 \mathrm{H})$ are highlighted using black lines. From these images, an appropriate quantification of the defect density for TMD epitaxy is obtained. Figure $4 \mathrm{c}$ represents our current optimal homoepitaxial nucleation and growth of $\mathrm{WSe}_{2}$ by PA-MBE. This growth was characterized by a nucleation density of $\sim 5 \mathrm{E}+10 \mathrm{~cm}^{-2}$ and a stacking control of $60 \% / 40 \%\left(3 \mathrm{R}_{22 \mathrm{H}}\right)$. The lower limit of the equivalent areal defect density is correspondingly calculated as large as $2.6 \mathrm{E}+10 \mathrm{~cm}^{-2}$.

Two possible routes to improve the homoepitaxy and hence lower the defect density are extracted from the graph presented in Figure $4 \mathrm{~b}$. The first route relies on improving the control on the bilayer stacking phase ( $3 \mathrm{R}$ versus $2 \mathrm{H})$. The increased control on the bilayer stacking phase (from $60_{/ 40}$ to $90_{/ 10}$ ) reduces the areal defect density to $3.2 \mathrm{E}+09 \mathrm{~cm}^{-2}$. This improvement is visually represented in Figure $4 \mathrm{~d}$. The second route relies on decreasing the nucleation density of the TMD epitaxy. This is visually represented in Figure 4e. Here, the nucleation density is reduced by 2 orders of magnitude to a value of $\sim 5 \mathrm{E}+08 \mathrm{~cm}^{-2}$ as compared to the nucleation density represented in Figure $4 \mathrm{c}$. This results in a reduction of the areal defect density down to $2.9 \mathrm{E}+08 \mathrm{~cm}^{-2}$.

Although both the improvement on the stacking control and the reduction of the nucleation density reduces the defect density for TMD epitaxy, according to our model, both routes are not equally promising. From Figure $4 b$, it is seen that the route relying on the improvement of the stacking control is only able to reduce the areal defect density by a factor 
$\sim 10$, which is only obtained when the bilayer stacking control is increased towards its upper boundary limits $\left(90_{/ 10}\right)$. Taking into account that obtaining a stacking control of $90 / 10$ will be extremely challenging as a consequence of the very similar binding energies for the various bilayer stacked phases due to the weak vdW interlayer interactions as revealed from our $a b$ initio calculations, a factor-10-gain in areal defect density can be considered as only marginal. Only an almost complete control on the bilayer stacking phase $\left(\sim 100_{\sim 0}\right)$ is predicted to significantly reduce the equivalent areal defect density. Accordingly, this work reveals that the lack of complete control on the bilayer stacking phase is a fundamental limitation of lowlydefective TMD vdW epitaxy.

To overcome this limitation, we emphasize the second route that relies on the reduction of the nucleation density of the vdW epitaxy. Here, the model reveals that when the nucleation density is reduced by a factor 10 , the areal defect density also reduces by a factor $\sim 10$. Since we believe the window for improvement on the nucleation density of TMD epitaxy is still relatively large, this route is highlighted to be more promising and is believed to be key towards a lower-defective TMD vdW epitaxy. Counterintuitively, the defect density of homoepitaxially grown TMDs, is thus highly dependent on its nucleation density.

Lastly, one can argue if the in-plane epitaxial seeding of TMDs for their integration on large-area substrates is still essential or not? Indeed, our work highlights that complete control on bilayer stacking is anyhow extremely challenging and therefore has only a marginal gain on the defect density. Consequently, the epitaxy of TMDs will always be characterized by a high density of stacking faults as a result of weak vdW interlayer coupling, which will eliminate the beneficial effect of the in-plane epitaxial seeding by the formation of $60^{\circ} \mathrm{GBs}$. Depending on the requirements of the targeted application, the gain of the in-plane epitaxial seeding can become marginal and other approaches such as selective nucleation by surface engineering ${ }^{47}$ or textured deposition directly on Silicon substrates ${ }^{48}$ might become preferred.

\section{CONCLUSION}

The vdW homoepitaxy was investigated in-depth by performing PA-MBE nucleation and growth of $\mathrm{WSe}_{2}$ on single-crystalline $\mathrm{WSe}_{2}$ exfoliated flakes. We found that the vdW homoepitaxy of the TMD compound was characterized by a high density of stacking faults, similarly as the observation of $60^{\circ}$ twins as for the case of vdW heteroepitaxy. The presence of the stacking faults was assigned to the too subtle differences in binding energy between the different bilayer stacking configurations as a result of the weak vdW interlayer interactions as explained by our ab-initio calculations. Two different bilayer stacking phases (3R and $2 \mathrm{H})$ 
were found to coexist simultaneously. Remarkably, the 3R:AB and 3R:AC bilayer stacking configurations were found not to be equally distributed which was explained by the presence of Se vacancies at the homoepitaxial growth surface. The coexistence of both the $3 \mathrm{R}$ and $2 \mathrm{H}$ bilayer stacking phases resulted in a high density of defective $60^{\circ} \mathrm{GBs}$ upon closure of the ML, which was extracted from our generalized epitaxial nucleation and growth model. This model was developed to quantify a lower limit of the defect density for TMD epitaxy in function of the nucleation density and the degree of control on the bilayer stacking phase $(3 \mathrm{R} / 2 \mathrm{H})$. The careful analysis of the outcomes of this model has highlighted that the inevitable presence of stacking faults is the main and fundamental limitation of lowly-defective TMD vdW epitaxy. This might open a potential for other synthesis approaches of vdW materials that reach beyond classical epitaxy.

\section{EXPERIMENTAL SECTION}

\subsection{The virtual $2 \mathrm{D}$ crystal substrate}

See Supporting Information.

\subsection{PA-MBE growth of $\mathrm{WSe}_{2}$}

$\mathrm{The}_{\mathrm{WSe}}$ is nucleated and grown using the technique of Plasma-Assisted Molecular Beam Epitaxy (PA-MBE) $)^{21,34}$. A W atomic flux generated from an electron-beam evaporator and a metallic Se atomic flux generated from a $\mathrm{H}_{2} \mathrm{Se}$ radio frequency plasma cell are simultaneously introduced in the growth chamber to form the stoichiometric $\mathrm{WSe}_{2}$ crystals. The growth rate is set at $0.15 \mathrm{ML} \mathrm{h}^{-1}$ which corresponds to a $\mathrm{W}$ flux of $0.16 \mathrm{E}+15$ atoms $\mathrm{cm}^{-2} \mathrm{~h}^{-1}$. The partial pressure of the selenium plasma is set at 2.0E-05 Torr, corresponding to a flow rate of $24 \mathrm{sccm}$. The growth temperature is optimum at $450{ }^{\circ} \mathrm{C}$.

\subsection{WSe 2 flake exfoliation, pick-up and transfer}

$\mathrm{The}_{\mathrm{WSe}}$ bulk crystals are supplied by HQ Graphene and have a purity of $>99.995 \%$. The flakes are exfoliated onto the thermally oxidized 90 -nm-thick $\mathrm{SiO}_{2}$ dielectric of the 2-inch $\mathrm{Si}$ substrates. For the TEM analysis, directly after the $\mathrm{WSe}_{2}$ homoepitaxy, the flakes are ex-situ picked-up and transferred to the TEM-grid according to the procedure described in the reference ${ }^{49}$. 


\subsection{Plan-view HR-TEM characterization}

A Titan3 G2 60-300 (FEI) with high brightness XFEG electron source is used for the HR-TEM analysis. The electron source is operated at $120 \mathrm{kV}$. SAEDPs are obtained using a beam diameter on the image plane of $\sim 780 \mathrm{~nm}$. Quantifoil TEM-grids having $2 \mu \mathrm{m}$ diameter holes at a pitch of $4 \mu \mathrm{m}$ are used.

\subsection{AFM characterization}

AFM images are taken with a Bruker Dimension at a scan rate of $0.6 \mathrm{~Hz}$ and with a resolution of $512 \times 512$ pixels per image.

\subsection{DFT calculations}

The ab-initio calculations are performed using DFT, as implemented in the SIESTA code ${ }^{50}$. The generalized gradient approximation (GGA) is used for the exchange-correlation functional, as parametrized by Perdew-Burke-Ernzerhof ${ }^{51}$. Core electrons are described by norm-conserving pseudopotentials ${ }^{52}$ and valence electrons are described using double-zeta polarized basis sets. The energy cut-off is fixed to $350 \mathrm{Ry}$, and a $(4 \times 4 \times 1)$ and $(20 \times 20 \times 1) \mathrm{k}-$ point mesh is used for the structural relaxation and binding energy calculations, respectively, with a convergence threshold for the residual atomic forces fixed to $0.01 \mathrm{eV} \AA^{-1}$. Long-range van der Waals interactions are included by using the DFT-D2 Grimme dispersion corrections ${ }^{53}$. The computations are performed on $(4 \times 4 \times 1) W_{2}$ supercells with a vacuum layer of approximately $15 \AA$ to avoid spurious interactions between the neighboring cells.

\subsection{Defect density calculation}

The $60^{\circ} \mathrm{GB}$ defect densities are calculated from $3.0 \mu \mathrm{m} \times 3.0 \mu \mathrm{m}$ simulations. The presented linear defect densities (expressed per $\mathrm{cm}$ ) are calculated from the average of three linear defect densities measured along the three symmetrical $\langle 1 \overline{1} 00\rangle$ directions. These linear defect densities are individually quantified as the average of 20 linear defect densities that are calculated using 20 contours separated by $0.12 \mu \mathrm{m}$ with a length of $2.4 \mu \mathrm{m}$. Additionally, the averaged linear defect densities are squared towards equivalent areal defect densities expressed per $\mathrm{cm}^{2}$. For improved statistics, every simulation is repeated 10 times. These repeated simulations have various but uniform nucleation sites in agreement with their corresponding nucleation density and control on bilayer stacking phase. 


\section{REFERENCES}

1. Novoselov KS, Geim AK, Morozov S V., et al. Electric field in atomically thin carbon films. Science (80- ). 2004;306(5696):666-669. doi:10.1126/science.1102896

2. Xia F, Mueller T, Lin Y, Valdes-Garcia A, Avouris P. Ultrafast graphene photodetector. Nat Nanotechnol. 2009;4(12):839-843. doi:10.1038/nnano.2009.292

3. Cheng R, Jiang S, Chen Y, et al. Few-layer molybdenum disulfide transistors and circuits for high-speed flexible electronics. Nat Commun. 2014;5:5143. doi:10.1038/ncomms6143

4. Kolobov A V. TMD Book. https://link-springercom.prox.lib.ncsu.edu/content/pdf/10.1007\%2F978-3-319-31450-1.pdf.

5. Song X, Guo Z, Zhang Q, Zhou P, Bao W, Zhang DW. Progress of Large-Scale Synthesis and Electronic Device Application of Two-Dimensional Transition Metal Dichalcogenides. Small. 2017;13(35):1-22. doi:10.1002/smll.201700098

6. Splendiani A, Sun L, Zhang Y, et al. Emerging photoluminescence in monolayer MoS2. Nano Lett. 2010;10(4):1271-1275. doi:10.1021/n1903868w

7. Zhang Y, Chang T-R, Zhou B, et al. Direct observation of the transition from indirect to direct bandgap in atomically thin epitaxial MoSe2. Nat Nanotechnol. 2014;9(2):111-115. doi:10.1038/nnano.2013.277

8. Garcia AGF, Neumann M, Amet F, et al. Effective cleaning of hexagonal boron nitride for graphene devices. Nano Lett. 2012;12(9):4449-4454. doi:10.1021/n13011726

9. Jariwala D, Sangwan VK, Lauhon LJ, Marks TJ, Hersam MC. Emerging device applications for semiconducting two-dimensional transition metal dichalcogenides. ACS Nano. 2014;8(2):1102-1120. doi:10.1021/nn500064s

10. Sarkar D, Xie X, Liu W, et al. A subthermionic tunnel field-effect transistor with an atomically thin channel. Nature. 2015;526(7571):91-95. doi:10.1038/nature15387

11. Li MO, Esseni D, Nahas JJ, Jena D, Xing HG. Two-dimensional heterojunction interlayer tunneling field effect transistors (Thin-TFETs). IEEE J Electron Devices Soc. 2015;3(3):200-207. doi:10.1109/JEDS.2015.2390643

12. Tsipas P, Fragkos S, Tsoutsou D, et al. Direct Observation at Room Temperature of the Orthorhombic Weyl Semimetal Phase in Thin Epitaxial MoTe2. Adv Funct Mater. 2018;28(33):1-12. doi:10.1002/adfm.201802084

13. Fragkos S, Sant R, Alvarez C, et al. Room Temperature Commensurate Charge Density Wave in Epitaxial Strained TiTe 2 Multilayer Films. Adv Mater Interfaces. 2019;6(5):1-9. doi:10.1002/admi.201801850 
14. Koma A. Van der Waals epitaxy for highly lattice-mismatched systems. J Cryst Growth. 1999;201:236-241. doi:10.1016/S0022-0248(98)01329-3

15. Barton AT, Yue R, Anwar S, et al. Transition metal dichalcogenide and hexagonal boron nitride heterostructures grown by molecular beam epitaxy. Microelectron Eng. 2015;147:306-309. doi:10.1016/j.mee.2015.04.105

16. Liu Y, Weiss NO, Duan X, Cheng HC, Huang Y, Duan X. Van der Waals heterostructures and devices. Nat Rev Mater. 2016;1(9). doi:10.1038/natrevmats.2016.42

17. Wang R, Lange FRL, Cecchi S, Hanke M, Wuttig M, Calarco R. 2D or Not 2D: Strain Tuning in Weakly Coupled Heterostructures. Adv Funct Mater. 2018;28(14):1-7. doi:10.1002/adfm.201705901

18. Boschker JE, Galves LA, Flissikowski T, Lopes JMJ, Riechert H, Calarco R. Coincident-site lattice matching during van der Waals epitaxy. Sci Rep. 2015;5(1):1-8. doi:10.1038/srep18079

19. Wang Y, Nakano M, Kashiwabara Y, Matsuoka H, Iwasa Y. Transport properties of a few nanometer-thick TiSe2 films grown by molecular-beam epitaxy. Appl Phys Lett. 2018;113(7):1-5. doi:10.1063/1.5039493

20. Suenaga K, Ji HG, Lin YC, et al. Surface-Mediated Aligned Growth of Monolayer MoS2 and In-Plane Heterostructures with Graphene on Sapphire. ACS Nano. 2018;12:10032-10044. doi:10.1021/acsnano.8b04612

21. Mortelmans W, El Kazzi S, Nalin Mehta A, et al. Peculiar alignment and strain of 2D WSe2 grown by van der Waals epitaxy on reconstructed sapphire surfaces . Nanotechnology. 2019;30(46):465601. doi:10.1088/1361-6528/ab3c9b

22. Lin YC, Jariwala B, Bersch BM, et al. Realizing Large-Scale, Electronic-Grade TwoDimensional Semiconductors. ACS Nano. 2018;12(2):965-975. doi:10.1021/acsnano.7b07059

23. Aminalragia-Giamini S, Marquez-Velasco J, Tsipas P, Tsoutsou D, Renaud G, Dimoulas A. Molecular beam epitaxy of thin HfTe2 semimetal films. 2D Mater. 2016;4(1):015001. doi:10.1088/2053-1583/4/1/015001

24. Nakano M, Wang Y, Kashiwabara Y, Matsuoka H, Iwasa Y. Layer-by-Layer Epitaxial Growth of Scalable WSe2 on Sapphire by Molecular Beam Epitaxy. Nano Lett. 2017;17(9):5595-5599. doi:10.1021/acs.nanolett.7b02420 
25. Ji Q, Kan M, Zhang Y, et al. Unravelling orientation distribution and merging behavior of monolayer MoS 2 domains on sapphire. Nano Lett. 2015;15(1):198-205. doi:10.1021/n1503373x

26. Dumcenco D, Ovchinnikov D, Marinov K, et al. Large-area epitaxial monolayer MoS2. ACS Nano. 2015;9(4):4611-4620. doi:10.1021/acsnano.5b01281

27. Zhang X, Choudhury TH, Chubarov M, et al. Diffusion-Controlled Epitaxy of Large Area Coalesced WSe2 Monolayers on Sapphire. Nano Lett. 2018;18(2):1049-1056. doi:10.1021/acs.nanolett.7b04521

28. Ma Y, Kolekar S, Coy Diaz H, et al. Metallic Twin Grain Boundaries Embedded in MoSe2 Monolayers Grown by Molecular Beam Epitaxy. ACS Nano. 2017;11(5):51305139. doi:10.1021/acsnano.7b02172

29. Vergnaud C, Dau M, Marty A, Jamet M. New approach for the molecular beam epitaxy growth of scalable single- crystalline WSe2 monolayers. arXiv:1910.08097 [cond-mat.mtrl-sci]

30. Chen L, Liu B, Ge M, Ma Y, Abbas AN, Zhou C. Step-Edge-Guided Nucleation and Growth of Aligned WSe2 on Sapphire via a Layer-over-Layer Growth Mode. ACS Nano. 2015;9(8):8368-8375. doi:10.1021/acsnano.5b03043

31. Zallo E, Cecchi S, Boschker JE, et al. Modulation of van der Waals and classical epitaxy induced by strain at the Si step edges in GeSbTe alloys. Sci Rep. 2017;7(1):14. doi:10.1038/s41598-017-01502-Z

32. Tarakina N V., Schreyeck S, Luysberg M, et al. Suppressing Twin Formation in Bi2Se3 Thin Films. Adv Mater Interfaces. 2014;1(5):1-8. doi:10.1002/admi.201400134

33. Levy I, Garcia TA, Shafique S, Tamargo MC. Reduced twinning and surface roughness of $\mathrm{Bi} 2 \mathrm{Se} 3$ and $\mathrm{Bi} 2 \mathrm{Te} 3$ layers grown by molecular beam epitaxy on sapphire substrates . J Vac Sci Technol B, Nanotechnol Microelectron Mater Process Meas Phenom. 2018;36(2):02D107. doi:10.1116/1.5017977

34. El Kazzi S, Mortelmans W, Nuytten T, et al. MoS2 synthesis by gas source MBE for transition metal dichalcogenides integration on large scale substrates. $J$ Appl Phys. 2018;123(13):135702. doi:10.1063/1.5008933

35. Yue R, Nie Y, Walsh LA, et al. Nucleation and growth of WSe2 : enabling large grain transition metal dichalcogenides. 2D Mater. 2017;4(4):1-11. doi:10.1088/20531583/aa8ab5 
36. Zhao X, Ding Z, Chen J, et al. Strain Modulation by van der Waals Coupling in Bilayer Transition Metal Dichalcogenide. ACS Nano. 2018;12(2):1940-1948. doi:10.1021/acsnano.7b09029

37. Vishwanath S, Liu X, Rouvimov S, et al. Comprehensive structural and optical characterization of MBE grown $\mathrm{MoSe}_{2}$ on graphite, $\mathrm{CaF}_{2}$ and graphene. 2 D Mater. 2015;2(2):024007. doi:10.1088/2053-1583/2/2/024007

38. Yue R, Barton AT, Zhu H, et al. HfSe 2 Thin Films : 2D Transition Metal Dichalcogenides Grown by Molecular Beam Epitaxy. ACS Nano. 2015;9(1):474-480. doi:10.1021/nn5056496

39. Dau MT, Gay M, Di Felice D, et al. Beyond van der Waals Interaction: The Case of MoSe2 Epitaxially Grown on Few-Layer Graphene. ACS Nano. 2018;12(3):23192331. doi:10.1021/acsnano.7b07446

40. Puretzky AA, Liang L, Li X, et al. Low-Frequency Raman Fingerprints of TwoDimensional Metal Dichalcogenide Layer Stacking Configurations. ACS Nano. 2015;9(6):6333-6342. doi:10.1021/acsnano.5b01884

41. Lin Z, Carvalho BR, Kahn E, et al. Defect engineering of two-dimensional transition metal dichalcogenides. 2D Mater. 2016;3(2). doi:10.1088/2053-1583/3/2/022002

42. Li W, Wang T, Dai X, et al. Bandgap engineering of different stacking WS2 bilayer under an external electric field. Solid State Commun. 2016;225:32-37. doi:10.1016/j.ssc.2015.10.013

43. Hong J, Wang Y, Wang A, et al. Atomistic dynamics of sulfur-deficient highsymmetry grain boundaries in molybdenum disulfide. Nanoscale. 2017;9(29):1031210320. doi:10.1039/c7nr02941a

44. Yan A, Ong CS, Qiu DY, et al. Dynamics of Symmetry-Breaking Stacking Boundaries in Bilayer MoS2. J Phys Chem C. 2017;121(40):22559-22566. doi:10.1021/acs.jpcc. $7 \mathrm{~b} 08398$

45. Komsa HP, Krasheninnikov A V. Engineering the Electronic Properties of TwoDimensional Transition Metal Dichalcogenides by Introducing Mirror Twin Boundaries. Adv Electron Mater. 2017;3(6):1-10. doi:10.1002/aelm.201600468

46. Ly TH, Perello DJ, Zhao J, et al. Misorientation-angle-dependent electrical transport across molybdenum disulfide grain boundaries. Nat Commun. 2016;7:1-7. doi:10.1038/ncomms10426 
47. Heyne MH, De Marneffe JF, Delabie A, et al. Two-dimensional WS2 nanoribbon deposition by conversion of pre-patterned amorphous silicon. Nanotechnology. 2017;28(4). doi:10.1088/1361-6528/aa510c

48. Huyghebaert C, Schram T, Smets Q, et al. 2D materials: Roadmap to CMOS integration. Tech Dig - Int Electron Devices Meet IEDM. 2019;2018-Decem:22.1.122.1.4. doi:10.1109/IEDM.2018.8614679

49. Roy T, Tosun M, Kang JS, et al. Field-effect transistors built from all two-dimensional material components. ACS Nano. 2014;8(6):6259-6264. doi:10.1021/nn501723y

50. Soler JM, Artacho E, Gale JD, et al. The SIESTA method for ab initio order-N materials simulation. J Phys Condens Matter. 2002;14(11):2745-2779. doi:10.1088/0953-8984/14/11/302

51. Perdew JP, Burke K, Ernzerhof M. Generalized gradient approximation made simple. Phys Rev Lett. 1996;77(18):3865-3868. doi:10.1103/PhysRevLett.77.3865

52. Troullier N, Martins JL. Efficient pseudopotentials for plane-wave calculations. Phys Rev B. 1991;43(3):1993-2006. doi:10.1103/PhysRevB.43.1993

53. STEFAN GRIMME. Semiempirical GGA-Type Density Functional Constructed with a Long-Range Dispersion Correction. Comput Chem. 2006;27:1787-1799. doi:DOI $10.1002 /$ jcc. 20495 